\title{
小麦谷氨酰胺合成酶同工酶转录特点及其启动子序列分析
}

\author{
王小纯 ${ }^{1,2}$ 王露露 ${ }^{1}$ 张志勇 $^{1}$ 秦步坛 $^{1}$ 于美琴 $^{2}$ 韦一昊 $^{1}$ \\ 马新明 1 ,
}

1 河南粮食作物协同创新中心 / 河南农业大学, 河南郑州 $450002 ;{ }^{2}$ 河南农业大学生命科学学院, 河南郑州 450002

摘 要: 谷氨酰胺合成酶是小麦氮同化关键酶, 分为胞液型和质体型 $(\mathrm{TaGS} 2)$ 两类, 其中胞液型 $\mathrm{TaGS}$ 又分为 $\mathrm{TaGS} 1$ 、 TaGSr 和 TaGSe。为了研究异源六倍体小麦 A、B、D 染色体组 TaGS 同工酶表达差异及调控机制, 本项目利用三代测 序技术测定了 TaGS 同工酶基因转录水平，依据中国春基因组序列克隆了豫麦 49 的 12 个 TaGS 同工酶启动子，并对其 序列进行了分析。结果表明, TaGS1 主要由 6B 染色体基因转录, TaGSe 和 TaGSr 主要由 4D 染色体基因转录, TaGS2 主 要由 $2 \mathrm{D}$ 染色体基因转录, 不同 TaGS 同工酶转录起始位点距起始密码子 ATG 的距离不同。启动子元件分析显示, 6B 染 色体上的 TaGS1 启动子有较多 W-box、AC-I、ABRE、as-1 和茉莉酸甲酯等响应元件, 4D 染色体上的 TaGSe 启动子有 较多胁迫响应转录因子(MYB、MBS、LTR 等)结合元件和植物生长素响应元件, 4D 染色体上的 TaGSr 启动子有较多 WRE3 等转录因子结合元件, 2D 染色体上的 TaGS2 启动子有较多 A-box、WRE3、ARE 及 AT 富集区。不同 TaGS 同工 酶启动子顺式元件种类、数目及排列顺序均不同, 为进一步研究 GS 同工酶调控机制奠定了基础。

关键词: 小麦; GS 同工酶; 转录; 启动子; 顺式作用元件

\section{Transcription characteristics of wheat glutamine synthetase isoforms and the sequence analysis of their promoters}

\author{
WANG Xiao-Chun ${ }^{1,2}$, WANG Lu-Lu ${ }^{1}$, ZHANG Zhi-Yong ${ }^{1}$, QIN Bu-Tan ${ }^{1}$, YU Mei-Qin ${ }^{2}$, WEI Yi-Hao ${ }^{1}$, and \\ MA Xin-Ming ${ }^{1, *}$ \\ ${ }^{1}$ Collaborative Innovation Center of Henan Grain Crops, Henan Agricultural University, Zhengzhou 450002, Henan, China; ${ }^{2}$ College of Life Science, \\ Henan Agricultural University, Zhengzhou 450002, Henan, China
}

\begin{abstract}
As a key enzyme for nitrogen assimilation in wheat, glutamine synthetase is grouped into two classes: cytosolic GS and chloroplastic GS (TaGS2), and cytosolic GS includes TaGS1, TaGSr, and TaGSe. In order to study the expression characteristics and regulatory mechanisms of GS isozymes in chromosome A, B, and D of heterohexaploid wheat, transcripts of TaGS isoforms were analyzed based on the third-generation sequencing technology transcriptome analysis, and 12 promoters of TaGS isozymes of Yumai 49 were cloned based on Chinese Spring genome, and the sequence of the promoters were analyzed. The results showed that TaGS1 was mainly transcribed on chromosome 6B, TaGSe and TaGSr on chromosome 4D, and TaGS2 on chromosome 2D. Furthermore, the distance from initiation codon ATG to initiation site of transcript for each promoter of TaGS was distinct. Promoter element analysis showed that the promoter of TaGS1 in 6B had more W-box, AC-I, ABRE, as-1, and methyl jasmonic response elements, the promoter of TaGSe in 4D had more stress response elements (MYB, MBS, LTR, etc.) and auxin response element, the promoter of TaGSr in 4D had more WRE3 and transcript factor response elements, the promoter of TaGS2 in 2D had more A-box, WRE3, ARE, and an AT enrichment region. In summary, the number, type and order of cis-elements of different promoters of TaGS isozymes were distinct, which provided the foundation for further study on the regulation mechanism of TaGS isozymes.
\end{abstract}

Keywords: wheat; GS isoforms; transcription; promoter; cis-element

\footnotetext{
本研究由国家重点研发计划项目(2016YFD0300205)和河南省现代农业产业技术体系项目(S2010-01-G04)资助。

This study was supported by the National Key Research and Development Program of China (2016YFD0300205) and the Modern Agricultural Technology System in Henan Province (S2010-01-G04).

*通信作者(Corresponding author): 马新明, E-mail: xinmingma@126.com

第一作者联系方式: E-mail: xiaochun.w@163.com

Received (收稿日期): 2020-06-03; Accepted (接受日期): 2020-10-17; Published online (网络出版日期): 2020-11-02.

URL: https://kns.cnki.net/kcms/detail/11.1809.S.20201030.1707.010.html
} 
氮是植物生长发育的必需元素, 在作物产量与品质 形成过程中起重要作用。谷氨酰胺合成酶 $(\mathrm{GS})$ 是植物氮素 同化和运转的关键酶 ${ }^{[1]}$, 根据亚细胞定位可分为胞液型 $(\mathrm{GS} 1)$ 和质体型 $(\mathrm{GS} 2)$ 两类 ${ }^{[2]}$, 小麦 $\mathrm{GS} 1$ 包含 $\mathrm{GS} 1.1$ (GS1)、 $\mathrm{GS} 1.2(\mathrm{GSr})$ 和 GS1.3 (GSe)。GS2 参与硝酸还原氨与光呼 吸释放氨同化, GS1 参与叶片衰老过程中氮素的再利用 ${ }^{[3]}$, $\mathrm{GSr}$ 参与根部系氮素同化运转, $\mathrm{GSe}$ 参与籽粒发育过程中 氮素利用 ${ }^{[4]}$ 。GS 活性与小麦籽粒可溶性蛋白含量及产量 密切相关 ${ }^{[5-9]}$ 。水稻过表达 GS1 基因, 其 GS 活性及可溶 性蛋白含量显著提高 ${ }^{[10]}$, 过表达 GS2 可以增强水稻的光 呼吸和光合作用强度, 水稻过表达 GS2 和烟草过表达

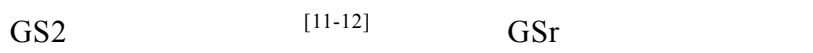
干旱及冷胁迫的敏感性 ${ }^{[13]}$ 。QTL 定位证明 GS 与小麦产量 呈正相关 ${ }^{[6]}$ 。可见, GS 与植物生长发育和抗逆密切相关。

小麦是异源六倍体, 有 $\mathrm{A} 、 \mathrm{~B} 、 \mathrm{D}$ 三个染色体组, 每 组染色体都有一套 $\mathrm{TaGS}$ 同工酶基因, 比如 GS1 有 3 个基 因编码, 即 $G S 1 A 、 G S 1 B$ 和 $G S 1 D$ 。以前报道小麦 GSr 和 $\mathrm{GSe}$ 只有 2 个基因 ${ }^{[14]}$, 可能存在未发现的 GSr 和 GSe 同 工酶基因。启动子位于基因的 $5^{\prime}$ 端非编码区, 其结构和功 能影响基因的转录调控。Michael 等 ${ }^{[15]}$ 发现拟南芥 GLN1:2 启动子在高氮条件下活性更高。拟南芥不同 GS 启动子存 在组织特异性, 与 GS 同工酶在植物中表达部位相吻 合 ${ }^{[16-17]}$ 。Gómez-Maldonado 等 ${ }^{[18]}$ 研究发现, 松树 GS 启动 子可与 MYB 转录因子结合提高 GS 同工酶的表达量从而 调控体内氮代谢。小麦染色体组复杂, 不同染色体组基因 表达存在差异。董佳敏等 ${ }^{[19]}$ 发现 A、B、D 染色体上的 expansin (膨胀素)基因均有表达, 胁迫处理后 $\mathrm{B}$ 染色体上 的 expansin 表达量最高, D 染色体表达量最低, 可见不同 染色体上的启动子调控方式不同。

有关植物 GS 功能及分布等研究报道较多, 但对 GS 启动子研究相对较少, 小麦 GS 启动子研究至今尚未见报 道。本研究依据中国春基因组序列, 在 A、B、D 染色体 上克隆 12 个 $\mathrm{GS}$ 同工酶启动子, 利用 Plant-CARE 在线软 件对其进行序列分析及功能预测, 利用三代测序技术测 定了小麦不同染色体组上 GS 同工酶的转录水平, 为深入 研究小麦 A、B、D 染色体组上不同 GS 同工酶表达调控 机制奠定了基础。

\section{1 材料与方法}

\section{1 试验材料}

试验品种为具有丰产稳产特点、氮素利用效率高的河 南省当家品种之一, 豫麦 49 (YM49)。将成熟饱满大小均 匀一致的小麦种子放入清水中浸泡, 露白后分别移入 Hoagland 营养液中进行培养至三叶一心, 分别取其根系 和叶片, 液氮处理后于 $-80^{\circ} \mathrm{C}$ 冰箱保存。许昌实验农场大 田种植的豫麦 49, 于 2018 年 10 月 16 日机械播种, 播种 前一次施入氮素量为 $225 \mathrm{~kg} \mathrm{hm}^{-2}$, 其他管理同大田。于小 麦开花 $16 \mathrm{~d}$ 后分别选取有代表性的旗叶、穗下节及籽粒,
每个处理重复 3 次, 液氮处理后于 $-80^{\circ} \mathrm{C}$ 冰箱保存。

\section{2 三代测序}

将上述所取的叶、穗下节、籽粒、根系样品分别于液 氮中研磨成粉状, 送百迈客生物科技公司, 由公司分别提 取各部位 RNA 并等量混合后测序。

\subsection{TaGS 同工酶基因查找}

依据本实验室前期克隆的小麦 GS 同工酶的 cDNA 序 列 [GenBank 号分别为: TaGS1:1 (DQ124209), TaGS1:2 (AY491968), TaGS1:3 (AY491970)和 TaGS2 (DQ124212)], 在中国春小麦基因组文库 (http://plants.ensembl.org/ Triticum_aestivum/Info/Index)中查找小麦 GS 基因。利用 ClustalX 软件进行序列对比, 确定编码 GS 同工酶的基因, 预测上游的启动子位置。

\section{4 引物设计}

以 1.3 查找的中国春 $\mathrm{TaGS}$ 同工酶启动子序列为模板, 利用 Premier 5.0 设计扩增目的片段的引物。上游引物(S) 在 ATG 上游 $2000 \mathrm{bp}$ 左右, 下游引物(A)在 ATG 下游 500 bp 左右(表 1)。扩增目的序列包含启动子及 ORF 的 5'端, 由华大基因合成引物。

\section{5 豫麦 49 基因组提取及启动子克隆}

利用 CTAB 法 ${ }^{[20]}$ 提取 YM49 基因组 DNA。以 YM49 基因组 DNA 为模板进行 PCR: $95^{\circ} \mathrm{C}$ 预变性 $180 \mathrm{~s} ; 95^{\circ} \mathrm{C}$ 变 性 $15 \mathrm{~s}$, 退火 $15 \mathrm{~s}$ (退火温度详见表 1 ), $72^{\circ} \mathrm{C}$ 延伸 $160 \mathrm{~s}$, 循 环 35 次; $72^{\circ} \mathrm{C}$ 稳定 $5 \mathrm{~min}^{\circ} \mathrm{PCR}$ 产物经 $1 \%$ 琼脂糖凝胶电 泳后, 切取与目的片段大小一致的条带, 利用胶回收试剂 盒(诺唯赞, DC301)回收目的条带, 然后与 pTOPO 载体(艾 德莱, DR01)连接, 转化 DH5 $\alpha$ 大肠杆菌, 利用卡那抗性平 板篮选阳性克隆, 进行 PCR 验证后送生工生物工程(上海) 股份有限公司测序。

\section{6 启动子序列分析}

测序后利用 ClustalX 软件与中国春小麦 GS 基因进行 序列对比, 并利用在线软件 Plant-CARE (http://bioinformatics. psb.ugent.be/webtools/plantcare/html/) 对启动子序列进行 顺式作用元件分析。

\section{7 转录起始位点预测}

将三代测序的 TaGS 同工酶 mRNA 序列 5'端 A/G 定 为转录起始位点; 若 5 端没有 $\mathrm{A} / \mathrm{G}$, 沿克隆的启动子序列 上游寻找最近的 $\mathrm{A} / \mathrm{G}$ 定为转录起始位点。根据克隆的启 动子序列, 距转录起始位点最近的 TATA-box 为 RNA 聚合 酶 II 识别位点 ${ }^{[21]}$ 。

\section{2 结果与分析}

\section{$2.1 \mathrm{TaGS}$ 同工酶在小麦染色体中表达特点}

$\mathrm{TaGS}$ 同工酶种类多, 且同源性高, 利用 $\mathrm{qPCR}$ 不能准 确反应各染色体上 TaGS 的表达情况。三代测序可以获得 YM49 的 TaGS 同工酶全长转录本, 便于准确区分各个 TaGS 基因。为了得到小麦所有 TaGS 同工酶表达信息, 选 择花后 $16 \mathrm{~d}$ 根系、节间、叶片、籽粒及苗期根系与叶片 
表 1 TaGS 同工酶启动子扩增引物

Table 1 Primers of TaGS isoenzymes for promoter amplification

\begin{tabular}{|c|c|c|c|c|}
\hline $\begin{array}{l}\text { 引物名称 } \\
\text { Primer name }\end{array}$ & $\begin{array}{c}\text { 引物序列 } \\
\text { Primer sequence }\left(5^{\prime}-3^{\prime}\right)\end{array}$ & $\begin{array}{c}\text { 退火温度 } \\
\text { Annealing temperature }\left({ }^{\circ} \mathrm{C}\right)\end{array}$ & $\begin{array}{c}\text { PCR 长度 } \\
\text { PCR fragment (bp) }\end{array}$ & $\begin{array}{l}\text { 5'端非编码区 } \\
5^{\prime} \mathrm{NCS}(\mathrm{bp})\end{array}$ \\
\hline GS1-6AL S & CACTGCCTTCTCAGGCTTGTTA & 58 & 2000 & 1594 \\
\hline GS1-6AL A & GCCCCATGAATTTAGCATCG & & & \\
\hline GS1-6BL S & TCTTAGTCCAACGTGGCATCATAGG & 62 & 2033 & 1548 \\
\hline GS1-6BL A & AGACGCCAAACTACGACTACG & & & \\
\hline GS1-6DL S & CACATTCACACGTGGTTTCCTCT & 58 & 2261 & 1470 \\
\hline GS1-6DL A & GCAAAAACCTCTTCTCGTCGC & & & \\
\hline GSe-4AL S & TCACCCATAATCATGCTGTGCAAAT & 63 & 2538 & 1557 \\
\hline GSe-4AL A & TACCATATGTACTCGGCGATGATCTTG & & & \\
\hline GSe-4BL A & ATGGGAGGTTGGGACAGTGC & 58 & 2508 & 1565 \\
\hline GSe-4BL $S$ & GATGCGAAGCATACGCGC & & & \\
\hline GSe-4DS S & TCTGAAGATGCTCATGAGTTGCTCAAT & 62 & 2536 & 1557 \\
\hline GSe-4DS A & TACCATATGTACTCGGCGATGATCTTG & & & \\
\hline GSr-4AS S & GTAATGTGGCTACGGTGTGAGTCTGTT & 61 & 2155 & 1548 \\
\hline GSr-4AS A & GGGTGCAGAATGCAAGTAGC & & & \\
\hline GSr-4BL S & TCATCTCCGTGAGGAGGTCTTGA & 62 & 2308 & 1615 \\
\hline GSr-4BL A & TACTCGACGATGATCTTGTCGGTG & & & \\
\hline GSr-4DL $S$ & GCCCGTTGTACCACTGTTATCGAT & 62 & 1624 & 1467 \\
\hline GSr-4DL A & TACTCGACGATGATCTTGTCGGTG & & & \\
\hline GS2-2AL S & GCATTCCTGGCTTGCATGTCTAGA & 58 & 2008 & 1499 \\
\hline GS2-2AL A & ATGATCTTGTCGGTGAAGGGC & & & \\
\hline GS2-2BL S & ATTTCGCCGAGTTCAGGTCGAG & 63 & 2153 & 1419 \\
\hline GS2-2BL A & ATGATCTTGTCGGTGAAGGGC & & & \\
\hline GS2-2DL S & CAGCGATCCTAGCCTTCGAAAAG & 60 & 1878 & 1097 \\
\hline GS2-2DL A & ATGATCTTGTCGGTGAAGGGC & & & \\
\hline
\end{tabular}

组织 RNA 等量混合测序。三代测序数据分析显示, 不同 染色体组的 TaGS 同工酶表达量存在差异。其中 TaGSr 基 因表达量最高, TaGSe 基因表达量最低。TaGS1 位于 6 号 染色体长臂 $(\mathrm{L})$, 在 $\mathrm{A} 、 \mathrm{~B} 、 \mathrm{D}$ 染色体上的表达量依次为 $6 \mathrm{~B}>$ $6 \mathrm{~A}>6 \mathrm{D} ; \mathrm{TaGSe}$ 和 $\mathrm{TaGSr}$ 位于 4 号 $\mathrm{B} 、 \mathrm{D}$ 染色体长臂 $(\mathrm{L})$ 或 A 染色体短臂 $(\mathrm{S})$ 。 $\mathrm{TaGSe}$ 在不同染色体上的表达量依次
为 $4 \mathrm{D}>4 \mathrm{~A}>4 \mathrm{~B} ; \mathrm{TaGSr}$ 在 $\mathrm{A} 、 \mathrm{~B} 、 \mathrm{D}$ 染色体上的表达量依次 则为 $4 \mathrm{D}>4 \mathrm{~B}>4 \mathrm{~A} ; \mathrm{TaGS} 2$ 位于 2 号染色体长臂 $(\mathrm{L})$, 在 $\mathrm{A}$ 、 $B 、 D$ 染色体上的表达量依次为 $2 \mathrm{D}>2 \mathrm{~B}>2 \mathrm{~A}$ (图 1)。

\subsection{TaGS 同工酶启动子克隆}

TaGS 同工酶基因转录受其启动子元件调控, 为了探 索其基因表达调控机制, 对 A、B、D 染色体上的 TaGS 同工酶

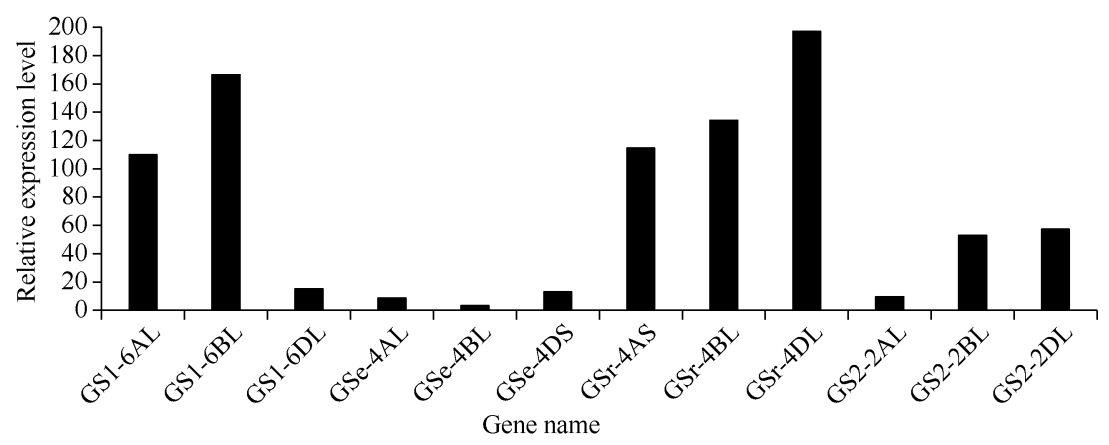

图 1 不同染色体上 TaGS 同工酶基因表达特点

Fig. 1 Expression of TaGS isozymes on different chromosomes 
启动子进行了克隆。首先以 YM49 基因组为模板, 利用特异 性引物(表 1)进行 PCR 扩增, 分别扩增到 A、B、D 染色体上 的 TaGS1、TaGSr、TaGSe 及 TaGS2 基因的启动子片段(图 2)。

GS1
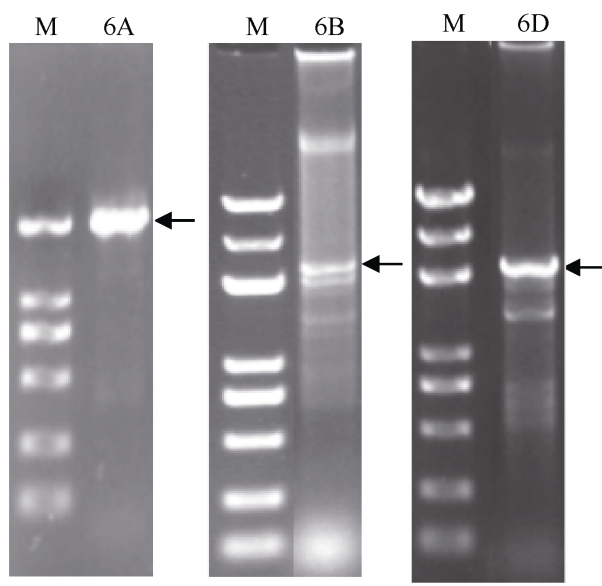

GSe

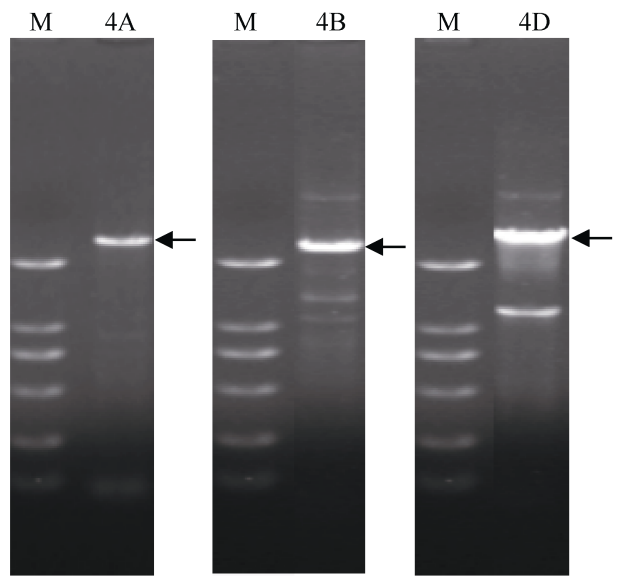

将与表 1 一致的目的片段回收, 与 $\mathrm{pTOPO}$ 载体连接转化 $\mathrm{DH} 5 \alpha$, 提取质粒, 并进行 PCR 鉴定后(图 3), 相应菌株进行测序, 结果 表明 YM49 与中国春的 GS 启动子序列同源性为 $100 \%$ 。
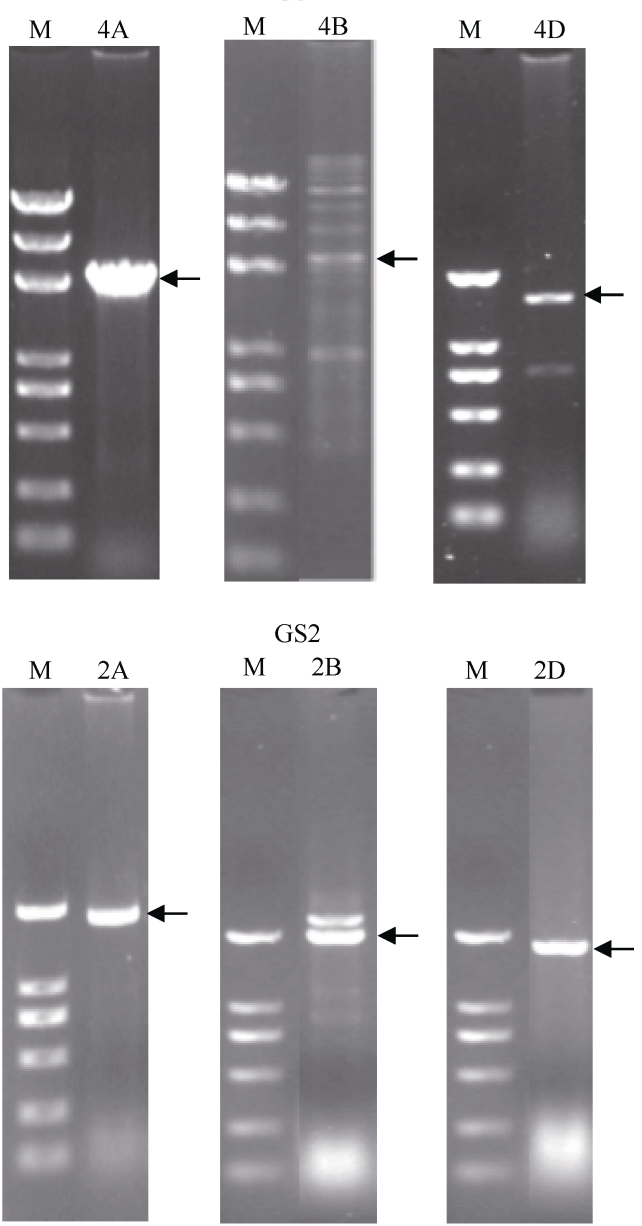

图 2 不同染色体 TaGS 同工酶启动子 PCR 扩增产物鉴定

Fig. 2 Identify PCR amplification of TaGS isoenzyme promoters on different chromosomes

箭头所指为目的片段。M: DNA 分子量标记。The arrows are the pointed to the target segment. M: marker.
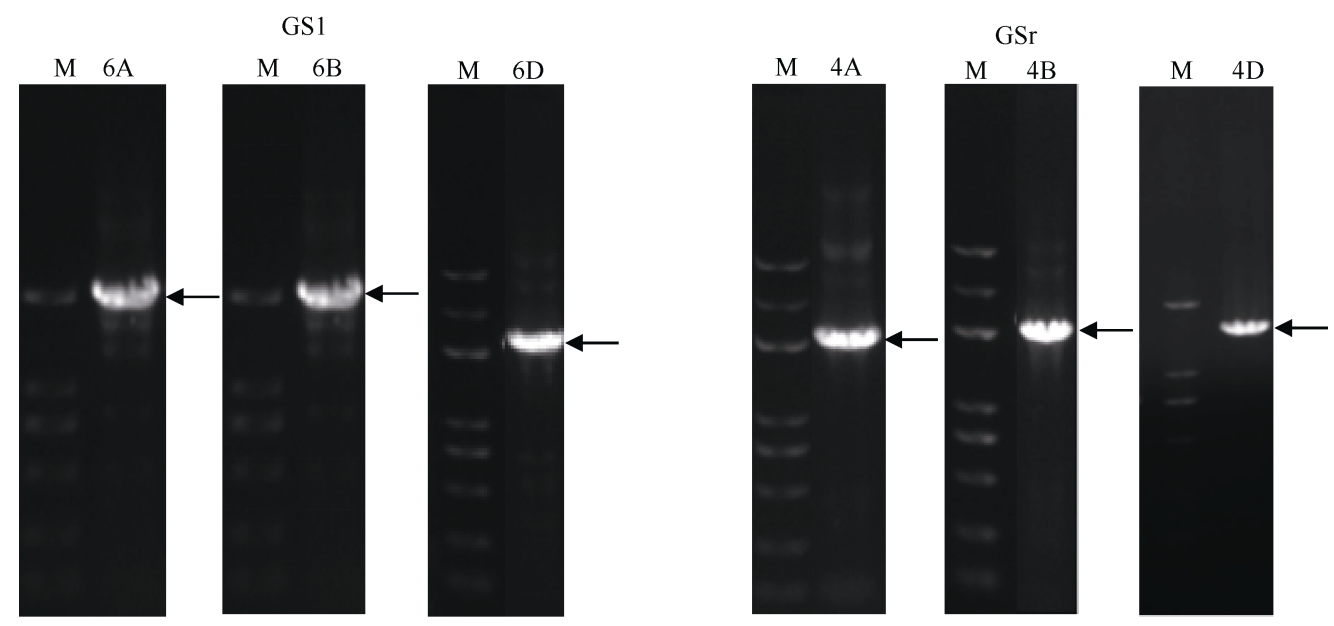

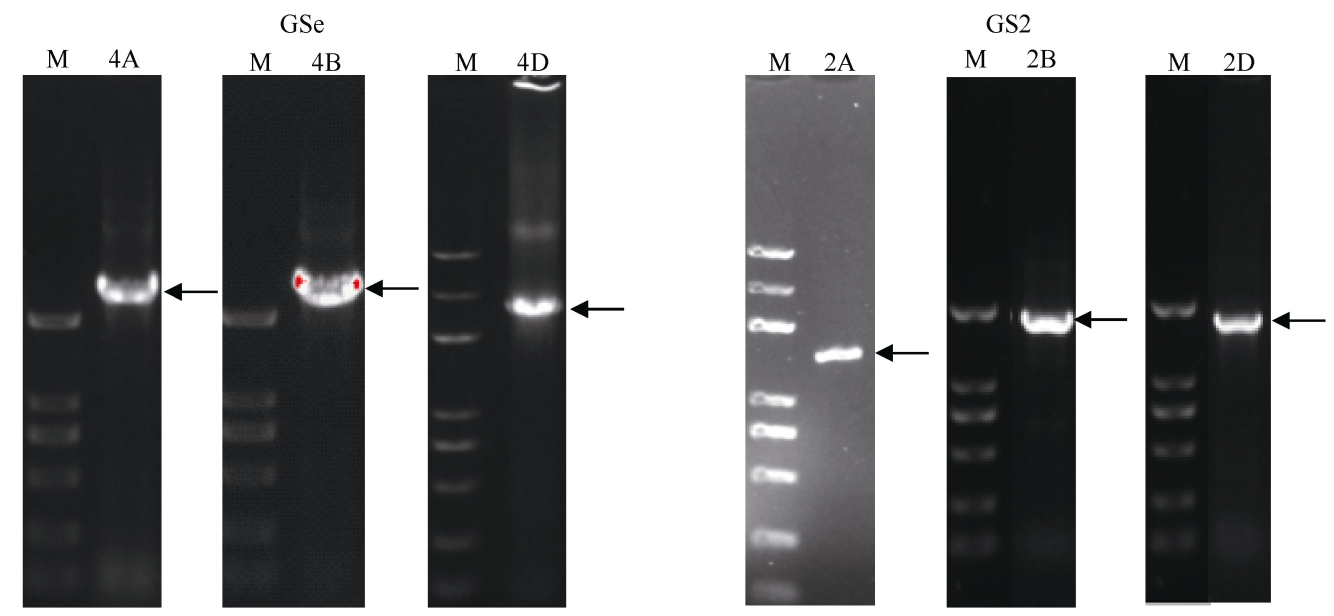

图 3 TaGS 同工酶启动子质粒 PCR 鉴定

Fig. 3 Identify recombinant plasmids of TaGS isoenzyme promoters by PCR

箭头所指为目的片段。 M: DNA 分子量标记。The arrows are the pointed to the target segment. M: marker.

\subsection{TaGS 同工酶启动子序列分析}

利用 Plant-CARE 在线软件对克隆测序的 12 个 TaGS 同工酶启动子进行分析，发现存在功能不同的多种顺式 元件(图 4)。包括基本起始元件 CAAT-box 和 TATA-box; 脱 落酸、水杨酸、生长素、赤霉素及茉莉酸等激素响应元件; 光响应元件; 厌氧、防御及低温等胁迫响应元件; 分生组 织表达调控元件及栅栏叶肉细胞分化元件等组织特异性 元件; 细胞周期及昼夜节律调节元件; MYB、MYC 等转录 因子结合位点，以及 as-1、W-box、dOCT 及 WRE3 等末 知功能响应元件。

不同 $\mathrm{TaGS}$ 同工酶启动子结构不同, 同一 $\mathrm{TaGS}$ 同工 酶在不同染色体上有其特殊的响应元件。6 $\mathrm{A}$ 染色体上的 TaGS1 启动子存在生长素响应元件和昼夜调控元件, $6 \mathrm{~B}$ 染色体上 TaGS1 启动子存在防御和胁迫响应元件, $6 \mathrm{D}$ 染 色体上 TaGS1 启动子存在 MYBHv1 结合位点。TaGSe 在 $4 \mathrm{~A}$ 染色体上存在干旱响应元件及 MYB 结合位点, 在 $4 \mathrm{~B}$ 染色体上存在 MYBHv1 结合位点、分生组织表达调控元 件, 在 4D 染色体上存在赤霉素响应元件、生长素响应元 件、低温响应元件、MYBHv1 结合位点、分生组织表达 调控元件。 $\mathrm{TaGSr}$ 在 $4 \mathrm{~A}$ 染色体上存在 A-box、MYC, 在 $4 \mathrm{~B}$ 染色体上存在赤霉素响应元件、水杨酸响应元件、缺 氧特异性诱导元件、细胞周期调控元件, 在 4D 染色体上 存在 MYBHv1 结合位点。TaGS2 在 $2 \mathrm{~A}$ 染色体上存在赤 霉素响应元件、厌氧诱导调控元件, 在 $2 \mathrm{~B}$ 染色体上存在 茉莉酸甲酯响应元件, 在 $2 \mathrm{D}$ 染色体上存在赤霉素响应元 件、茉莉酸甲酯响应元件、厌氧诱导调控元件。

基于 $\mathrm{TaGS}$ 同工酶启动子克隆与序列分析，结合三代 测序结果, 对不同 TaGS 同工酶基因的转录起始位点进行 了预测(图 5)。结果表明: 胞液型 TaGS(包括 TaGS1、TaGSe 和 TaGSr) 在 A、B、D 染色体上的转录起始位点位于 ATG 上游约-88 - $249 \mathrm{bp}$, 且不同染色体组之间存在差异。 TaGS1 位于 6 号染色体, 在 A、B、D 染色体上转录起始
位点分别位于 ATG 上游-202 bp、-249bp、-171 bp; TaGSe 和 TaGSr 位于 4 号染色体, TaGSe 在 A、B、D 染色体上 转录起始位点分别位于 ATG 上游-110 bp、-139 bp、-191 bp; TaGSr 在 A、B、D 染色体上转录起始位点分别位于 ATG 上游 $-88 \mathrm{bp} 、-89 \mathrm{bp} 、-107 \mathrm{bp}$ 。质体型 TaGS2 在 A、 B、D 染色体上的转录起始位点距 ATG 最远, 差异也最大, 约-111 -631 bp。TaGS2 位于 2 号染色体, 在 A、B、D 染色体上转录起始位点分别位于 ATG 上游-111 bp、 $-631 \mathrm{bp} 、-448 \mathrm{bp}$ 。

小麦不同 $\mathrm{TaGS}$ 同工酶启动子存在特异的响应元件, 不同的环境条件下、不同的生长发育时期、不同的组织器 官影响不同染色体上的 TaGS 同工酶转录水平不同, 而转 录本 5'端非编码区与翻译起始效率密切相关, 进一步影 响 TaGS 同工酶表达量及生物活性, 从而影响小麦的氮素 利用、产量和品质。

\section{3 讨论}

启动子是基因表达的开关，可分为组成型、组织特异 型和诱导型 3 种类型 ${ }^{[22]}$, 启动子上的顺式作用元件与基 因表达水平密切相关。前人研究表明, 顺式作用元件的种 类、数量、顺序及距离可能会影响转录的起始及转录水 平 ${ }^{[23-24]}$ 。启动子上游存在的增强子或抑制子可能增强或降 低基因的表达水平。栽培小麦不同染色体上的 TaGS 同工 酶启动子元件不同, 可能响应不同环境条件, 以确保 TaGS 表达水平，使小麦具有丰产稳产的特性。本实验室 前期 TaGS 同工酶活性测定及 Western 杂交表明花后 $16 \mathrm{~d}$ 小麦旗叶及子粒 GS 同工酶条带最多, 表达量也最大 ${ }^{[13]}$ 。 为了获得小麦 GS 同工酶转录本及转录量全面信息, 本研 究以豫麦 49 花后 $16 \mathrm{~d}$ 各组织器官及苗期叶片和根系为材 料, 采用三代测序技术研究了 TaGS 同工酶在不同染色体 上的表达特点, 发现豫麦 49 不同染色体的 GS1、GSr、 GSe 和 GS2 表达量不同, 但 GSr、GSe 和 GS2 都是 D 染 
色体基因表达量最大, D 组染色体使栽培小麦从二粒小麦 进化到现在的多粒小麦, 根系、叶片及籽粒主要 GS 同工 酶的大量表达是氮素同化的基础，也是产量形成的基础。

研究启动子的方法主要是生物信息学预测分析及实 验验证, 本文利用生物信息学对 $\mathrm{TaGS}$ 的 12 个启动子序列 进行分析, 发现每个启动子中存在多个 TATA-box, 结合 三代测序数据分析表明在 $\mathrm{A} / \mathrm{G}$ 上游可能只有 1 个 TATA-box 是 RNA 聚合酶 II 识别位点, 不同 TaGS 启动子 的起始转录位点与 TATA-box 距离差别又十分大, 可能意
味着起始转录的机理不尽相同; 每个启动子都有许多光 响应元件, 但是只有 TaGS2 酶表达量及活性受光照强度 影响 ${ }^{[13]}$, 当利用启动子与 $G U S$ 构建重组载体转化拟南芥 时, 发现只有 GS2-2AL 启动子受光诱导(结果尚未发表)。 Plant-CARE 在线分析还发现每个 GS 启动子除了基本调 控元件 CAAT-box 外, 还存在多种非生物胁迫应答元件, 如缺氧/厌氧调控元件、干旱响应原件、低温响应原件等 以及转录因子如 MYB、MYC 等结合位点, 启动子上的顺 式元件特点与基因功能密切相关 ${ }^{[25]}$ 。乙烯、茉莉酸和赤

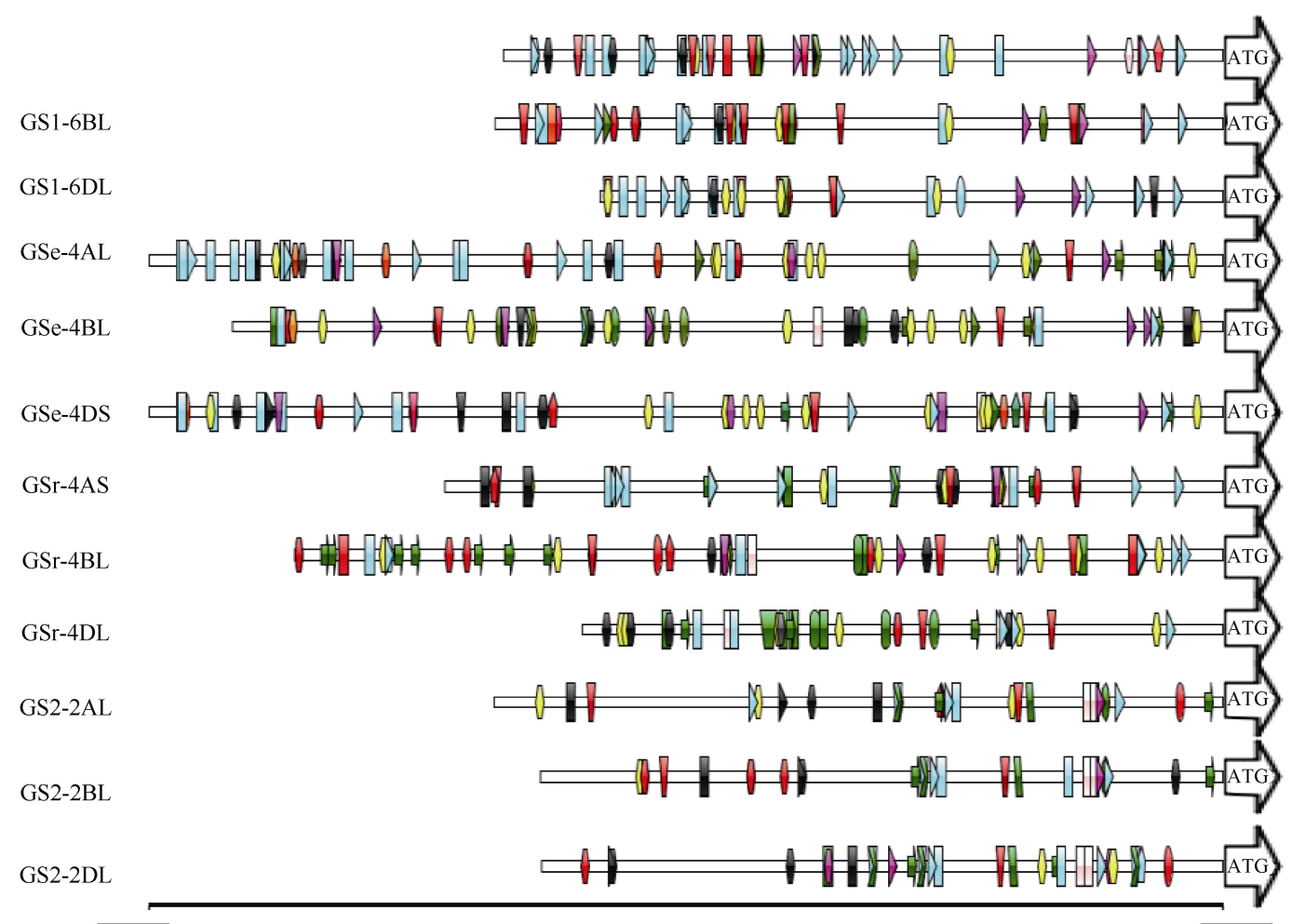

$-2538$

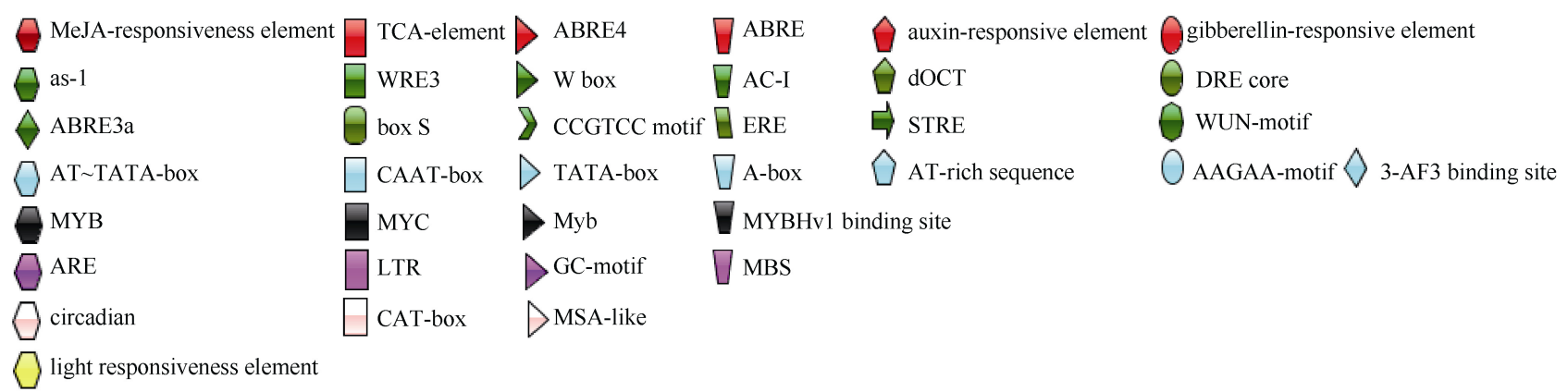

图 4 不同 TaGS 同工酶启动子顺式元件种类及分布

Fig. 4 Types and distribution of cis-elements in different TaGS isozyme promoters

不同颜色代表不同类型的响应元件, 颜色和形状代表一个具体的响应元件。红色: 激素响应元件; 绿色: 功能未知的响应元件; 蓝色: 基础元件; 黑色: 转录因子结合位点; 紫色: 胁迫响应元件; 粉色: 组织特异性响应元件; 黄色: 光响应元件。

Different colors represent different types of response elements; color and shape represent a specific response element. Red: hormone response element; Green: unknown function response element; Blue: basic element; Black: transcription factor binding site; Purple: stress response element; Pink: tissue specific response element; Yellow: light response element. 


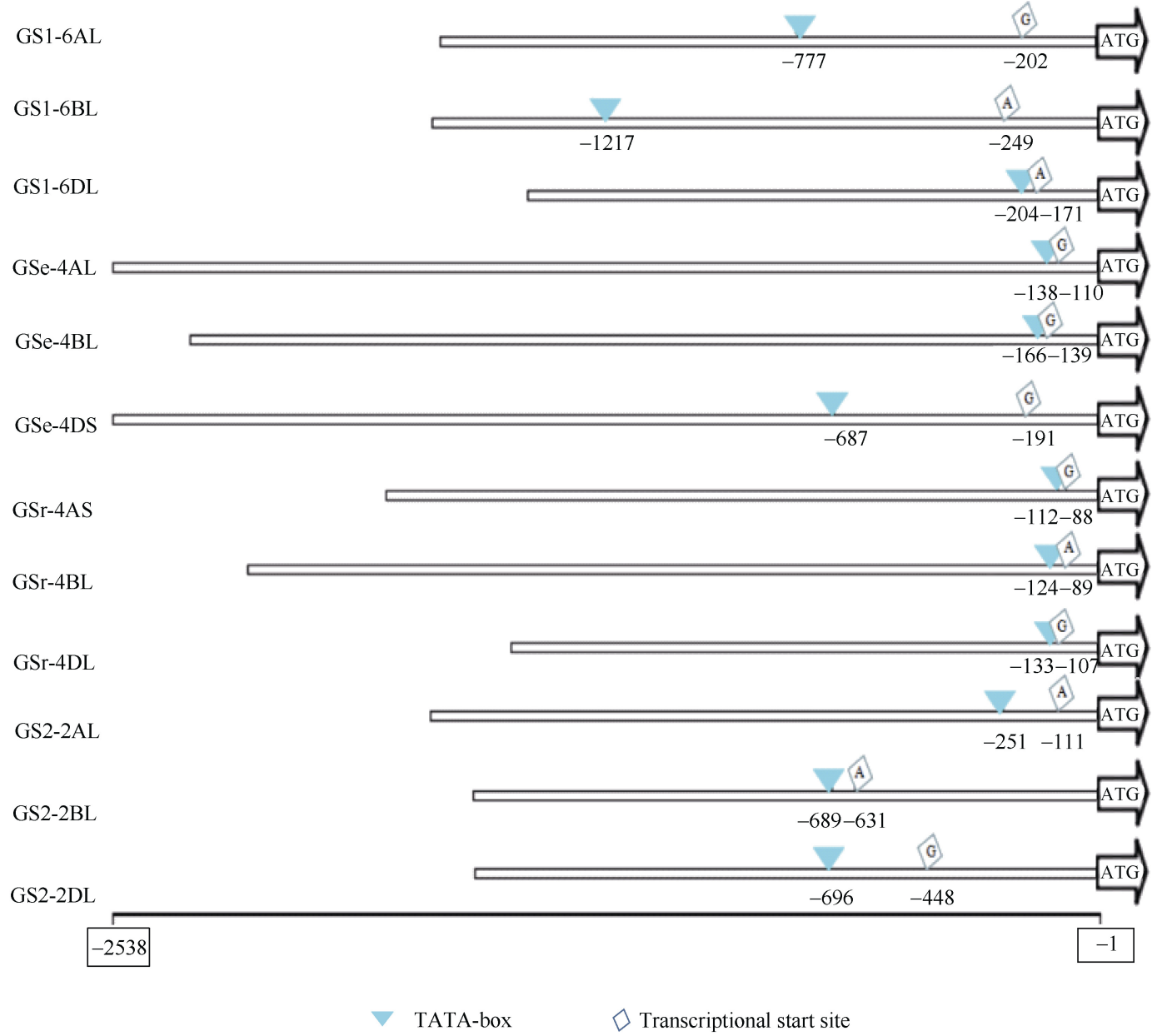

图 5 不同 TaGS 同工酶转录起始位点预测

Fig. 5 Prediction of transcription initiation site of different TaGS isozyme

TATA-box 为 RNA 聚合酶 II 识别位点。TATA box is the RNA polymerase II recognition site.

霉素处理使大豆 GmWRIIa 基因启动子活性增强 ${ }^{[26]}$, 干旱 可诱导小麦 $T a G A P C p 1$ 启动子活性 ${ }^{[27]}$, 小麦 $T a S A P 1$ 基因 启动子响应干旱、低温及 $\mathrm{ABA}{ }^{[28]}$, 松树的 GS 启动子可 与 MYB 转录因子结合从而提高 GS 同工酶的表达量 ${ }^{[18]}$ 等。TaGS 同工酶启动子元件数量及种类不同, 对外界环 境应答不同, 可能是 $\mathrm{TaGS}$ 同工酶差异表达及在 $\mathrm{A} 、 \mathrm{~B} 、 \mathrm{D}$ 染色体上差异表达的原因。但是这些启动子元件是否真正 存在, 哪个部位的响应元件起作用, 以及如何响应外界环 境的变化等还需要进一步验证。

\section{References}

[1] Miflin B J, Habash D Z. The role of glutamine synthetase and glutamate dehydrogenase in nitrogen assimilation and possibilities for improvement in the nitrogen utilization of crops. $J$ Exp Bot, 2002, 53: 979-987.

[2] Ortega J L, Wilson O L, Sengupta-Gopalan C. The 5' untranslated region of the soybean cytosolic glutamine synthetase $\beta 1$ gene contains prokaryotic translation initiation signals and acts as a translational enhancer in plants. Mol Genet Genom, 2012, 287: 881-893.
[3] Keys A J. The re-assimilation of ammonia produced by photorespiration and the nitrogen economy of $\mathrm{C}_{3}$ higher plants. Photosynth Res, 2006, 87: 165-175.

[4] Konishi N, Saito M, Imagawa F, Kanno K, Yamaya T, Kojima S. Cytosolic glutamine synthetase isozymes play redundant roles in ammonium assimilation under low-ammonium conditions in roots of Arabidopsis thaliana. Plant Cell Physiol, 2018, 59: 601-613.

[5] Kichey T, Heumez E, Pocholle D, Pageau K, Vanacker H, Dubois F, Gouis J L, Hirel B. Combined agronomic and physiological aspects of nitrogen management in wheat highlight a central role for glutamine synthetase. New Phytol, 2006, 169: 265-278.

[6] Li H M, Liang H, Li Z, Li Z, Tang Z X, Fu S L, Geng Y Y, Yan B J, Ren Z L. Dynamic QTL analysis of protein content and glutamine synthetase activity in re-combinant inbred wheat lines. Genet Mol Res, 2015, 14: 8706-8715.

[7] Kichey T, Hirel B, Heumez E, Dubois F, Le Gouis J. In winter wheat (Triticum aestivum L.) post-anthesis nitrogen uptake and remobilization to the grain correlates with agronomic traits and nitrogen physiological markers. Field Crops Res, 2007, 102: 22-32. 
[8] Bernard S M, Moller A L, Dionisio G, Kichey T, Jahn T P, Dubois F, Baudo M, Lopes M S, Tercé-Laforgue T, Foyer C H, Parry M A, Forde B G, Araus J L, Hirel B, Schjoerring J K, Habash D Z. Gene expression, cellular localization and function of glutamine synthetase isozymes in wheat (Triticum aestivum L.). Plant Mol Biol, 2008, 67: 89-105.

[9] 王小燕, 于振文. 不同小麦品种主要品质性状及相关酶活性 研究. 中国农业科学, 2005, 38: 1980-1988.

Wang X Y, Yu Z W. Study on main quality traits and related enzyme activities of different wheat varieties. Sci Agric Sin, 2005, 38: 1980-1988 (in Chinese with English abstract).

[10] Cai H, Zhou Y, Xiao J, Li X, Zhang Q, Lian X. Overexpressed glutamine synthetase gene modifies nitrogen metabolism and abiotic stress responses in rice. Plant Cell Rep, 2009, 28: 527-537.

[11] 贾喜婷, 韦一吴, 谷明金金, 石爱博, 王小纯. 过表达 $T a G S 1 / T a G S 2$ 对烟草抗盐能力的影响及其机制. 中国烟草学 报, 2017, 121: 112-117.

Jia X T, Wei Y H, Gu M X, Shi A B, Wang C H. Effects of overexpression of TaGS1/TaGS2 on tobacco salt resistance and its mechanism. Chin J Tob Sci, 2017, 121: 112-117 (in Chinese with English abstract).

[12] Hoshida H, Tanaka Y, Hibino T, Hayashi Y, Tanaka A, Takabe $\mathrm{T}$, Takabe T. Enhanced tolerance to salt stress in transgenic rice that overexpresses chloroplast glutamine synthetase. Plant $\mathrm{Mol}$ Biol, 2000, 43: 103-111.

[13] 张同勋. 小麦谷氨酰胺合成酶在氮素代谢中的功能分析. 河 南农业大学硕士学位论文, 河南郑州, 2012. pp 7-32.

Zhang T X. Functional Analysis of Wheat Glutamine Synthase in Nitrogen Metabolism. MS Thesis of Henan Agricultural University, Zhengzhou, Henan, China, 2012. pp 7-32 (in Chinese with English abstract).

[14] Céline M D, Michèle R C, Karine P, Maud L, Olivier G, Joceline K, Marie H V, Magali F, Tiphanie J, Akira S. Glutamine synthetase-glutamate synthase pathway and Glutamate dehydrogenase play distinct roles in the sink-source nitrogen cycle in tobacco. Plant Physiol, 2006, 140: 444-456.

[15] Moison M, Marmagne A, Dinant S, Soulay F, Azzopardi M, Lothier J, Citerne S, Morin H, Legay N, Chardon F, Avice J C, Reisdorf-Cren M, Masclaux-Daubresse C. Three cytosolic glutamine synthetase isoforms located in different order veins work together for $\mathrm{N}$ remobilization and seed filling in Arabidopsis. J Exp Bot, 2018, 69: 4379-4393.

[16] Tabuchi M, Sugiyama K, Ishiyama K, Inoue E, Sato T, Takahashi $H$, Yamaya T. Severe reduction in growth rate and grain filling of rice mutants lacking $O s G S 1: 1$, a cytosolic glutamine synthetase1:1. Plant J, 2005, 42: 641-651.

[17] Tabuchi M, Abiko T, Yamaya T. Assimilation of ammonium ions and reutilization of nitrogen in rice (Oryza sativa L.). J Exp Bot, 2007, 58: 2319-2327.

[18] Gómez-Maldonado J, Avila C, Torre F, Cañas R, Cánovas F M, Campbell M M. Functional interactions between a glutamine synthetase promoter and MYB proteins. Plant J, 2004, 39: 513-526.

[19] 董佳敏, 徐永清, 彭丽娜, 冯旭, 姚树宽, 赵巧芩, 李凤兰, 胡宝忠. 小麦品种东农冬麦 2 号根中 TaEXPA7 部分同源基因
的克隆及表达特性分析. 麦类作物学报, 2017, 37(11): 25-33. Dong J M, Xu Y Q, Peng L N, Feng X, Yao S K, Zhao Q C, Li L $\mathrm{F}, \mathrm{Hu} \mathrm{B}$ Z. Cloning and expression characteristics analysis of some homologous genes TaEXPA7 in root of winter wheat Dongnong No.2 variety. $J$ Wheat Crops, 2017, 37(11): 25-33 (in Chinese with English abstract).

[20] 何辉. 光皮桦 BlOFPs 基因克隆及其互作蛋白分析. 浙江农林 大学硕士学位论文, 浙江杭州, 2016. pp 15-76.

$\mathrm{He} \mathrm{H}$. Cloning of the BlOFPs Gene and Analysis of Its Interaction Proteins in Birch. MS Thesis of Zhejiang A \& F University, Hangzhou, Zhejiang, China, 2016. pp 15-76 (in Chinese with English abstract).

[21] 李志英, 牟红珍, 高丁梅, 丁国平, 马婷, 王盛. 本氏烟 I 型 启动子的克隆及其转录起始位点分析. 中国生物工程杂志, 2014, 34(1): 28-35.

Li Z Y, Mou H Z, Gao D M, Ding G P, Ma T, Wang S. Cloning of cigarette type I promoter and analysis of its transcriptional starting sites. Chin J Biol Eng, 2014, 34(1): 28-35 (in Chinese with English abstract).

[22] 李一琨, 王金发. 高等植物启动子研究进展. 植物学通报, 1998, 15(增刊 1): 1-6.

Li Y K, Wang J F. Research progress of higher plant promoters. Bot Bull, 1998, 15(S1): 1-6 (in Chinese with English abstract).

[23] 路静, 赵华燕, 何奕昆, 宋艳茹. 高等植物启动子及其应用研 究进展. 自然科学进展, 2004, 14: 856-862.

Lu J, Zhao H Y, He Y K, Song Y R. Advances in higher plant promoters and their applications. Adv Nat Sci, 2004, 14: 856-862 (in Chinese with English abstract).

[24] 王昊龙, 韩俊杰, 李卫华, 刘伟. 抗性淀粉含量不同的小麦品 种(系)淀粉分支酶 EIIn 和 EII6 基因多态性分析. 新疆农业科 学, 2015, 52: 981-987.

Wang H L, Han J J, Li W H, Liu W. Resistant starch content of different wheat varieties (or lines) of starch branching enzyme EIIn and EII6 gene polymorphism analysis. Xinjiang Agric Sci, 2015, 52: 981-987 (in Chinese with English abstract).

[25] 崀珩, 李昂, 刘惠民, 景蕊莲. 小麦蛋白磷酸酶 $2 \mathrm{~A}$ 基因 $T a P P 2 A b B{ }^{\prime \prime}-\alpha$ 启动子的克隆及表达分析. 作物学报, 2016, 42: 1282-1290.

Yi H, Li A, Liu H M, Jing R L. Wheat protein phosphatase 2A gene $T a P P 2 A b B "-\alpha$ promoter cloning and expression analysis. Acta Agron Sin, 2016, 42: 1282-1290 (in Chinese with English abstract).

[26] 邵宇鹏, 杨明明, 包格格, 孙英楠, 杨强, 李文滨, 王志坤. 大豆 GmWRII $a$ 基因启动子克隆及其功能分析. 中国油料作物 学报, 2019, 41: 517-523.

Shao Y P, Yang M M, Bao G G, Sun Y N, Yang Q, Li W B, Wang $\mathrm{Z}$ K. Cloning and functional analysis of soybean GmWRI1a promoter. Chin J Oil Crop Sci, 2019, 41: 517-523 (in Chinese with English abstract).

[27] 魏文杰, 邓霞, 杨淑慎. 非生物胁迫下小麦 $T a G A P C p 1$ 基因启 动子的功能分析. 福建师范大学学报(自然科学版), 2019, 35(2): 76-84.

Wei W J, Deng X, Yang S S. Functional analysis of TaGAPCp1 gene promoter in wheat under abiotic stress. J Fujian Norm Univ (Nat Sci Edn), 2019, 35(2): 76-84 (in Chinese with English abstract). 
[28] 常建忠, 董春林, 张正, 乔麟轶, 杨睿, 蒋丹, 张彦琴, 杨丽莉, 吴佳洁, 景惢莲. 小麦抗逆相关基因 TaSAP1 的 5'非翻译区含 子功能分析. 作物学报, 2019, 45: 1311-1318.

Chang J Z, Dong C L, Zhang Z, Qiao L Y, Yang R, Jiang D, Zhang Y Q, Yang L L, Wu J J, Jing R L. Functional analysis of 5' non-translational subdomains of stress-related gene TaSAP1 in wheat. Acta Agron Sin, 2019, 45: 1311-1318 (in Chinese with English abstract).
[29] 金正勋, 李丹, 李明月, 同拉嘎, 潘冬, 张玉磊, 王海微, 韩云 飞, 张忠臣. 水稻谷氨酰胺合成酶同工型基因转录表达量与启 动子结构关系分析. 东北农业大学学报, 2017, 48(10): 1-10.

Jin Z X, Li D, Li M Y, Tong L G, Pan D, Zhang Y L, Wang H W, Han Y F, Zhang Z C. Relationship between transcriptional expression of glutamine synthase isotype gene and promoter structure in rice. J Northeast Agric Univ, 2017, 48(10): 1-10 (in Chinese with English abstract). 